\title{
Tachyonic inflation in loop quantum cosmology
}

\author{
Kui Xiao ${ }^{\mathrm{a}}$ (1) \\ Department of Science of Mathematics and Physics and Technology of Energy, Hunan Institute of Technology, Hengyang 421002, China
}

Received: 13 August 2019 / Accepted: 6 December 2019 / Published online: 17 December 2019

(c) The Author(s) 2019

\begin{abstract}
A tachyon field might be responsible for cosmological inflation at an early time and contribute to cosmological dark matter at a later time. We investigate tachyonic inflation by analyzing a tachyon field with different potentials in the framework of loop quantum cosmology. No matter which tachyon field energy dominates at the bounce, the evolution of the background can be divided into three phases: super-inflation, damping, and slow-roll inflation. The duration of each phase depends on the initial condition. During the slow-roll inflation, when the initial condition is $V\left(T_{\mathrm{B}}\right) / \rho_{\mathrm{c}} \geq 10^{-6}$, the number of e-folds is very high $(N \gg 60)$ for $V \propto T^{-n}$ with $n=1$ and $1 / 2$. For an exponential potential, to get enough e-folds, $V\left(T_{\mathrm{B}}\right) / \rho_{\mathrm{c}}$ should be greater than $7.802 \times 10^{-4}$. Furthermore, the probability of slow-roll inflation is obtained. We find that the probability of obtaining slow-roll inflation with 60 or more e-folds is very close to 1 .
\end{abstract}

\section{Introduction}

The inflationary paradigm has solved many problems in the standard model of cosmology [1,2]. Cosmic inflation provides a means to generate primordial cosmological perturbations [3-5]. The spatial curvature of the universe seeds the observed anisotropies in the cosmic microwave background [6], and it describes the formation of the large-scale structure of the universe [7]. In a simple inflationary model, the universe is dominated by a scalar field whose potential energy dominates over the kinetic energy, and the Hubble rate is constant during this stage. Evidence shows inflationary theory is an excellent candidate for explaining the very early universe [6]. However, inflationary theory usually considers a quantum field propagating on a classical spacetime described by general relativity (GR), which requires considering the Big Bang singularity. This singularity problem

\footnotetext{
a e-mail: 87xiaokui@gmail.com
}

is considered as a defect of GR on the high-energy scale. One way to solve this singularity is the quantum theory of gravity.

There are many approaches to quantum gravity, and some candidates include bouncing regimes. One of these is loop quantum cosmology (LQC) [8-10]. LQC is a canonical quantization of homogeneous spacetimes based on techniques used in loop quantum gravity (LQG) [11-13]. The dynamical behavior of LQC can be studied effectively by introducing quantum gravitational corrections to the gravitational and matter Hamiltonians. In LQC, holonomic corrections lead to an additional term $-\rho^{2} / \rho_{\mathrm{c}}$ in the Friedmann equation at high energy [14], where $\rho_{\mathrm{c}}$ is the maximum matter energy, which is determined by the quantum effect. The Hubble parameter reaches zero as the matter energy density reaches its maximum $\rho=\rho_{\mathrm{c}}$, where the quantum bounce occurs. After the quantum bounce, the cosmology enters a superinflation stage dominated by the quantum effect because $1<\rho / \rho_{\mathrm{c}}<1 / 2$. The quantum-modified term in the Friedmann equation can be ignored when $\rho \ll \rho_{\text {c }}$, and the dynamical behavior of the cosmology is described by GR. The question whether the desired slow-roll inflation can occur after the quantum bounce in LQC has been widely discussed [1520]. The simplest inflation model is given by scalar field theory with slow-roll evolution. Inflation with a canonical scalar field has been investigated for various potentials such as quadratic [20-22], Starobinsky [22-26], monodromy [27], and power-law ( $\phi^{n}$ with $\left.n<2\right)$ [28] potentials [29]. Inflation with a non-canonical scalar field has also been discussed [3033]. By considering the pre-inflation dynamical behavior of the background, one finds that a kinetic-energy-dominated (KED) bouncing point is more suitable for getting enough e-foldings during slow-roll inflation regardless of the type of potential [21,25-28,34].

Tachyonic inflation is an attractive and popular $\mathrm{K}$-inflation model, where the inflation is achieved with a nonstandard kinetic term in the inflation Lagrangian. A tachyon condensate with an effective Dirac-Born-Infeld action is an inter- 
esting scalar field that might be responsible for cosmological inflation at an early time and contributes to cosmological dark matter at a later time $[35,36]$. The tachyon has been investigated as an inflaton in LQC [31-33].

In this paper, we investigate tachyonic inflation in LQC. Many studies have reported that canonical scalar field inflation more easily achieves enough e-folds at a KED bounce $[25-28,34]$. Consequently, our first goal is to investigate the duration of tachyon inflation considering three types of potentials: an exponential potential and inverse-power potentials with $T^{-1}$ and $T^{-1 / 2}$. We will discuss which initial condition gives enough e-folds. Second, we determine whether the conclusion that canonical scalar field inflation arises naturally [15-19] is still holding for a tachyon field.

This paper is organized as follows. In Sect. 2, we briefly introduce the basic equations of the background evolution of the universe in LQC. In Sect. 3, we examine the duration of tachyon inflation and discuss the three potentials. This section analyzes in detail the evolution of the background in the framework of a negative tachyon velocity $\dot{T}<0$ and in terms of the ratio between the potential $V(T)$ and the critical energy density $\rho_{\mathrm{c}}$. The tachyon inflation probability is presented in Sect. 4. The main conclusions are summarized in the last section.

\section{Background}

The effective equations in LQC are modified with the LQG correction. This provides a surprisingly good approximation of the dynamics of sharply peaked LQC states at all times, including the bounce point where the quantum effects are strongest [37-40]. In this section, we follow Ref. [41] to briefly introduce the modified Friedmann equation in LQC. To get the modified Friedmann equation, we consider the Hamilton equation

$\dot{v}=\frac{3}{\gamma \lambda} v \sin (\lambda b) \cos (\lambda b)$,

in which $v=4 \mathscr{V}_{0} a^{3} M_{\mathrm{Pl}}^{2} / \gamma$ is the volume of a fixed fiducial cubic cell, $b=-\gamma P_{(a)} /\left(6 a^{2} \mathscr{V}_{0} M_{\mathrm{Pl}}^{2}\right)$ is the conjugate momentum to $v, a$ is the scale factor, $P_{(a)}$ is the conjugate momentum to the scale factor, the Barbero-Immirzi parameter $\gamma \simeq 0.2375[42,43], \lambda=\sqrt{\sqrt{3} \gamma /\left(2 M_{\mathrm{Pl}^{2}}\right)}$ is a constant, and $M_{\mathrm{Pl}}=1 / \sqrt{8 \pi}$ is the reduced Planck mass. The "dot" refers to cosmic time.

The relationship between the Hubble parameter $H$ and $b$ is

$H=\frac{\dot{a}}{a}=\frac{1}{2 \gamma \lambda} \sin (2 \lambda b)$, and the energy density $\rho$ relates to $b$ via

$\frac{\sin ^{2}(\lambda b)}{\gamma^{2} \lambda^{2}}=\frac{1}{3 M_{\mathrm{Pl}}^{2}} \rho$.

Combining the above equation and Eq. (2), we get the modified Friedmann equation in LQC,

$H^{2}=\frac{\dot{v}^{2}}{9 v^{2}}=\frac{1}{3 M_{\mathrm{Pl}}^{2}} \rho\left(1-\frac{1}{\rho_{\mathrm{c}}}\right)$

with the critical energy density $\rho_{\mathrm{c}}=\frac{3}{8 \pi \gamma^{2} \lambda^{2}}=\frac{2 \sqrt{3}}{8 \pi \gamma^{3}}$.

Similarly, we get the modified Raychaudhuri equation,

$\frac{\ddot{a}}{a}=-\frac{1}{6 M_{\mathrm{Pl}^{2}}} \rho\left(1-4 \frac{\rho}{\rho_{\mathrm{c}}}\right)-\frac{1}{2 M_{\mathrm{Pl}}^{2}} P\left(1-\frac{2 \rho}{\rho_{\mathrm{c}}}\right)$.

Using the modified Friedmann equation (4) and the Raychaudhuri equation (5), we get the equation for the rate of change of the Hubble parameter:

$\dot{H}=-\frac{1}{2 M_{\mathrm{Pl}}^{2}}(\rho+P)\left(1-\frac{2 \rho}{\rho_{\mathrm{c}}}\right)$.

We consider the matter source as a single tachyon field $T$ with the Hamiltonian [35,36]

$\mathscr{H}=2 v \pi \gamma \ell_{\mathrm{Pl}}^{2} \sqrt{V(T)^{2}+\left(2 v \pi \gamma \ell_{\mathrm{Pl}}^{2}\right)^{-2} \Pi^{2}}$,

with the field potential $V(T)$. The energy density and the pressure are $[32,35,36]$

$\rho=\frac{V(T)}{\sqrt{1-\dot{T}^{2}}}, \quad P=-V(T) \sqrt{1-\dot{T}^{2}}$.

These equations immediately yield a matter conservation law:

$\dot{\rho}+3 H(\rho+P)=0$.

The conservation equation gives the Klein-Gordon equation for a tachyon field,

$\frac{\ddot{T}}{1-\dot{T}^{2}}+3 H \dot{T}+\frac{V^{\prime}}{V}=0$,

where the prime denotes the derivative with respect to the field. The modified Friedmann Eq. (4) shows the occurrence of a nonsingular quantum bounce. When $\rho=\rho_{\mathrm{c}}$, the Hubble parameter becomes zero and the quantum bounce occurs. After the bounce, the universe enters a super-inflation stage in which the Hubble parameter $H$ and scale factor $a$ increase very quickly. The super-inflation ends when $\rho=\rho_{\mathrm{c}} / 2$ and 
the Hubble parameter reaches its maximum value $H_{\max }=$ $\sqrt{\kappa \rho_{\mathrm{c}} / 12}$. Many researchers studying on the evolution of the background have found that the probability of slow-roll inflation is almost 1 [15-20].

The dynamical behavior of the tachyon field has been discussed by many authors [31-33,44]. Xiong and Zhu [32] found that the number of quantum e-folds is lower in the slow-roll limit than in the classical case, and sufficient tachyonic field inflation in LQC requires a higher constant $V_{0}$ or lower tachyon mass $\alpha$ for an exponential potential. The same results were obtained by Xiao et al. [33]. In this paper, we set $V_{0}=\rho_{\mathrm{c}}$ for simplicity, because $V(T) \leq \rho_{\mathrm{c}}$ at the bounce point.

\section{Tachyonic inflation}

When a potential $V(T)$ is specified, Eqs. (4), (5), and (10) can be solved numerically by specifying the initial values of $a, \dot{a}, T$, and $\dot{T}$ at a specific time. A convenient choice of such a time is the bounce $t=t_{\mathrm{B}}$, with the subscript " $\mathrm{B}$ " indicating the bounce point. The critical energy density $\rho_{\mathrm{c}}$ is a given constant and $\dot{a}_{\mathrm{B}}=0$, then the initial conditions $\left(a_{\mathrm{B}}, \dot{a}_{\mathrm{B}}, T_{\mathrm{B}}, \dot{T}_{\mathrm{B}}\right)$ of the differential Eqs. (4), (5), and (10) are reduced to $\left(a_{\mathrm{B}}, T_{\mathrm{B}}\right)$, because one can write $\dot{T}_{\mathrm{B}}$ in terms of $\rho_{\mathrm{c}}$ and $T_{\mathrm{B}}$. For simplicity, we set $a_{\mathrm{B}}=1$ at the bounce. In this case, the initial conditions are reduced to specify the value of $T_{\mathrm{B}}$ only. The initial values of $\dot{T}_{\mathrm{B}}$ have two classes, $\dot{T}_{\mathrm{B}}>0$ and $\dot{T}_{\mathrm{B}}<0$; we just consider $\dot{T}_{\mathrm{B}}<0$ in this paper.

To discuss slow-roll inflation, we first introduce the following background quantities:

(I) The ratio between $V\left(T_{\mathrm{B}}\right)$ and $\rho_{\mathrm{c}}$,

$$
F_{\mathrm{B}}=\frac{V\left(T_{\mathrm{B}}\right)}{\rho_{\mathrm{c}}} .
$$

The value of $F_{\mathrm{B}}$ depends on the initial conditions. Because $V\left(T_{\mathrm{B}}\right) \in\left(0, \rho_{\mathrm{c}}\right]$, then $F_{\mathrm{B}} \in(0,1]$. However, we consider $F_{\mathrm{B}} \in\left[10^{-6}, 1\right]$ for simplicity.

(II) The equation of state $(\mathrm{EoS})$, which is defined by

$\omega=\frac{P}{\rho}=-1+\dot{T}^{2}$.

During slow-roll inflation, $\omega=-1$. Considering Eqs. (8) and (11), we get

$\omega_{\mathrm{B}}=-F_{\mathrm{B}}^{2} \in\left[-1,-10^{-12}\right]$.

When $F_{\mathrm{B}} \gg 0.5$, the potential energy dominates the kinetic at the bounce, and we denote this as the potential-energy-dominated (PED) case while denoting $F_{\mathrm{B}} \ll 0.5$ as KED.

(III) The slow-roll parameters $\epsilon_{\mathrm{H}}$ and $\delta_{\mathrm{H}}$, which are defined by the derivative of the Hubble parameter,

$\varepsilon_{\mathrm{H}}=-\frac{\dot{H}}{H^{2}}, \quad \delta_{\mathrm{H}}=\frac{\ddot{H}}{2 \dot{H} H}$.

During slow-roll inflation, $\varepsilon_{\mathrm{H}}, \delta_{\mathrm{H}} \ll 1$.

(IV) The number of e-folds of slow-roll inflation, $N$, which is defined as

$N \equiv \ln \left(\frac{a_{\mathrm{end}}}{a_{\mathrm{on}}}\right)$,

in which the subscript "on/end" means the onset/end of slow-roll inflation. We define the onset of inflation as $\varepsilon_{\mathrm{H}}=0.01$ or $\delta_{\mathrm{H}}=0.01$ and the end of inflation as $\varepsilon_{\mathrm{H}}=1$.

In this paper, we will discuss slow-roll inflation by considering three typical potentials,

$$
V \in\left\{V_{0} \mathrm{e}^{-\alpha T}, V_{1} x^{-1}, V_{1 / 2} x^{-1 / 2}\right\}
$$

in which $\alpha, V_{1}$ and $V_{1 / 2}$ are constants determined by observational data. According to the Planck 2018 results [6], we get $\alpha=5.66 \times 10^{-3}, V_{1}=3.57 \times 10^{-3}$, and $V_{1 / 2}=7.12 \times 10^{-4}$.

We first focus on the evolution of the background. At the bounce point, the energy density $\rho_{\mathrm{B}}=\rho_{\mathrm{c}}$, and the Hubble parameter $H_{\mathrm{B}}=0$. Immediately after the bounce, the Hubble parameter $H$ grows to its maximum value $H_{\max }$. This is the super-inflation stage. When $H=H_{\max }, \dot{H}=0$, the superinflation ends. For a KED bounce, the duration of superinflation is about 0.8 Planck seconds, no matter what the form of the potential is. This is reasonable, because when the kinetic energy dominates, the potential term in Eqs. (4) and (10) can be ignored and the duration is independent of the potential. The duration of super-inflation for a PED bounce is about 0.8 Planck seconds for an inverse-power potential and about $10^{4}$ Planck seconds for an exponential one. When the bounce is PED, such as for $F_{\mathrm{B}}=1, \dot{T}_{\mathrm{B}}=0$, but $\ddot{T}_{\mathrm{B}} \simeq 116$ for $V_{1} T_{\mathrm{B}}^{-1}, \ddot{T}_{\mathrm{B}} \simeq 1.7 \times 10^{5}$ for $V_{1 / 2} T_{\mathrm{B}}^{-1 / 2}$, and $\ddot{T}_{\mathrm{B}} \simeq 0.02$ for an exponential potential. An inverse-power potential holds its value at first and then increases to its maximum $V_{\text {max }}$ ( $V_{\max }=\rho$, see the third plot of Figs. 2,3 ) during the superinflation stage. An exponential potential is approximately constant at first (until $\sim 10^{3}$ Planck seconds after the bounce) and then decreases slowly (from $\sim 10^{3}$ Planck seconds to $\sim 10^{5}$ Planck seconds) during the super-inflation stage (see the third plot in Fig. 1). The background enters a damping stage as soon as the super-inflation ends. The duration of the damping stage, $t_{\mathrm{dds}}$, depends on the type of potential and initial conditions. For example, when $F_{\mathrm{B}}=1, t_{\mathrm{dds}} \simeq 100$ 

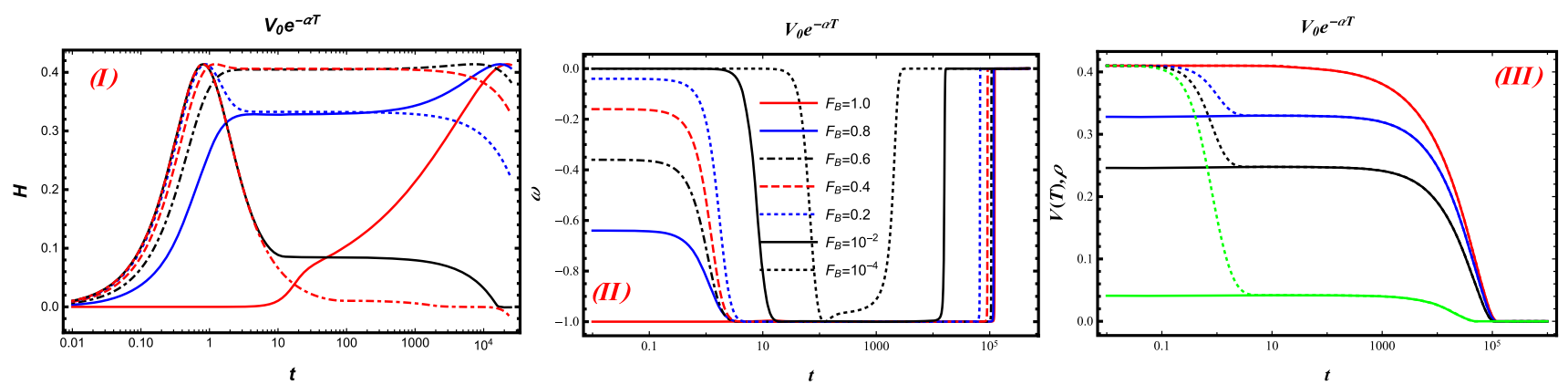

Fig. 1 Evolutions of $H, \omega, V(T)$, and $\rho$ for an exponential potential. The initial conditions for plots I and II are shown in II. Plot III is of $V(T)$ (solid) and $\rho$ (dotted) for the initial conditions $F_{\mathrm{B}}=1$ (red), $F_{\mathrm{B}}=0.8$ (blue), $F_{\mathrm{B}}=0.6$ (black), and $F_{\mathrm{B}}=0.1$ (green)

Table 1 Quantities involved in background evolution. The time $t_{\mathrm{si}}$ is the end of super-inflation, and $t_{\mathrm{on}}\left(t_{\mathrm{end}}\right)$ is the time of onset (end) of slow-roll inflation. The values of $F_{\mathrm{B}}$ start from the PED case and approach the KED one

\begin{tabular}{|c|c|c|c|c|c|c|c|}
\hline & $F_{\mathrm{B}}$ & $T_{\mathrm{B}}$ & $t_{\mathrm{si}} / t_{\mathrm{Pl}}$ & $t_{\mathrm{on}} / t_{\mathrm{Pl}}$ & $t_{\mathrm{end}} / t_{\mathrm{Pl}}$ & $\rho\left(T_{\text {end }}\right) / \rho_{\mathrm{c}}$ & $N$ \\
\hline \multirow[t]{12}{*}{$V_{0} \mathrm{e}^{-\alpha T}$} & 1 & 0 & $2.2 \times 10^{4}$ & $2.3 \times 10^{4}$ & $1.22 \times 10^{5}$ & $10^{-6}$ & $2.37 \times 10^{4}$ \\
\hline & 0.8 & 39.4038 & $1.7 \times 10^{4}$ & $1.8 \times 10^{4}$ & $1.17 \times 10^{5}$ & $10^{-6}$ & $2.36 \times 10^{4}$ \\
\hline & 0.6 & 90.2041 & $7.3 \times 10^{3}$ & $7.5 \times 10^{3}$ & $1.07 \times 10^{5}$ & $10^{-6}$ & $2.40 \times 10^{4}$ \\
\hline & 0.4 & 161.803 & 1.21222 & 10.0598 & $9.13 \times 10^{4}$ & $10^{-6}$ & $2.06 \times 10^{4}$ \\
\hline & $10^{-1}$ & 406.602 & 0.820886 & 16.3144 & $4.86 \times 10^{4}$ & $10^{-6}$ & $6.20 \times 10^{3}$ \\
\hline & $10^{-2}$ & 813.204 & 0.805562 & 44.7098 & $1.61 \times 10^{4}$ & $10^{-6}$ & 675.43 \\
\hline & $10^{-3}$ & 1219.81 & 0.805413 & 121.483 & $5.38 \times 10^{3}$ & $10^{-6}$ & 75.52 \\
\hline & $7.802 \times 10^{-4}$ & 1263.64 & 0.805409 & 134.964 & $4.81 \times 10^{3}$ & $10^{-6}$ & 60.00 \\
\hline & $10^{-4}$ & 1626.41 & 0.805409 & 303.509 & $2.08 \times 10^{3}$ & $10^{-6}$ & 9.41 \\
\hline & 1 & $8.605 \times 10^{-3}$ & 0.805412 & 59777.6 & $4.406 \times 10^{5}$ & $10^{-7}$ & 262.80 \\
\hline & $10^{-1}$ & $8.605 \times 10^{-2}$ & 0.805411 & 59769.8 & $4.367 \times 10^{5}$ & $10^{-7}$ & 260.72 \\
\hline & $10^{-2}$ & $8.064 \times 10^{-1}$ & 0.809675 & 59784.4 & $4.455 \times 10^{5}$ & $10^{-7}$ & 265.44 \\
\hline \multirow[t]{7}{*}{$V_{1} T^{-1}$} & $10^{-3}$ & 8.065 & 0.805413 & 59767.1 & $4.426 \times 10^{5}$ & $10^{-7}$ & 263.94 \\
\hline & $10^{-4}$ & $8.605 \times 10^{1}$ & 0.805411 & 59771.4 & $4.406 \times 10^{5}$ & $10^{-7}$ & 262.86 \\
\hline & $10^{-5}$ & $8.605 \times 10^{2}$ & 0.805411 & 62387 & $4.538 \times 10^{5}$ & $10^{-7}$ & 266.32 \\
\hline & $10^{-6}$ & $8.605 \times 10^{3}$ & 0.805217 & 6581.06 & $6.117 \times 10^{5}$ & $10^{-7}$ & 352.39 \\
\hline & 1 & $3.0169 \times 10^{-6}$ & 0.805463 & 8546.9 & $1.459 \times 10^{5}$ & $10^{-5}$ & 704.48 \\
\hline & $10^{-1}$ & $3.0169 \times 10^{-4}$ & 0.805478 & 8536.5 & $1.460 \times 10^{5}$ & $10^{-5}$ & 704.55 \\
\hline & $10^{-2}$ & $3.0169 \times 10^{-2}$ & 0.806278 & 8535.8 & $1.459 \times 10^{5}$ & $10^{-5}$ & 704.65 \\
\hline \multirow[t]{4}{*}{$V_{1 / 2} T^{-1 / 2}$} & $10^{-3}$ & 3.0169 & 0.805413 & 8536.1 & $1.459 \times 10^{5}$ & $10^{-5}$ & 704.44 \\
\hline & $10^{-4}$ & $3.0169 \times 10^{2}$ & 0.805411 & 8530.3 & $1.479 \times 10^{5}$ & $10^{-5}$ & 708.89 \\
\hline & $10^{-5}$ & $3.0169 \times 10^{4}$ & 0.805410 & 8268.1 & $4.188 \times 10^{5}$ & $10^{-6}$ & 881.22 \\
\hline & $10^{-6}$ & $3.0169 \times 10^{6}$ & 0.805814 & 13990.8 & $4.173 \times 10^{5}$ & $10^{-7}$ & 333.84 \\
\hline
\end{tabular}

Planck seconds for an exponential potential, $8.5 \times 10^{3}$ Planck seconds for $V_{1 / 2} T^{-1 / 2}$, and $5.9 \times 10^{4}$ Planck seconds for $V_{1} T^{-1}$ (see Table 1). The background enters the slow-roll inflation stage as the damping regime ends. After the slowroll inflation stage, the EoS tends to $\omega=0$, and the tachyon field contributes to cosmological dark matter (see the second plots of Figs. 1, 2, 3).

We consider slow-roll inflation as starting at $\delta_{\mathrm{H}}=0.01$ (because $\epsilon_{\mathrm{H}}$ reaches 0.01 before $\delta_{\mathrm{H}}$ ) and ending at $\epsilon_{\mathrm{H}}=1$. The numerical results are shown in Table 1. For the exponen- tial potential, the energy density $\rho\left(t_{\mathrm{on}}\right) \lesssim \rho_{\mathrm{c}} / 2$ for $F_{\mathrm{B}}=1$, $0.8,0.6$, and 0.4 , and $\rho\left(t_{\mathrm{on}}\right) \ll \rho_{\mathrm{c}}$ for $F_{\mathrm{B}} \leq 0.1$. For an inverse-power potential, $\rho\left(t_{\mathrm{on}}\right) \ll \rho_{\mathrm{c}}$ always held for all considering $F_{\mathrm{B}}$, the slow-roll inflation occurs in the classical region. When the slow-roll inflation ends, $\rho / \rho_{\mathrm{c}} \lesssim 10^{-5}$ for all three potentials and the quantum modification term can be ignored.

We consider that there is at least 60 e-folds during the slow-roll inflation. Just as shown in Table 1, the e-folds of tachyonic inflation depends on the type of potential and initial 

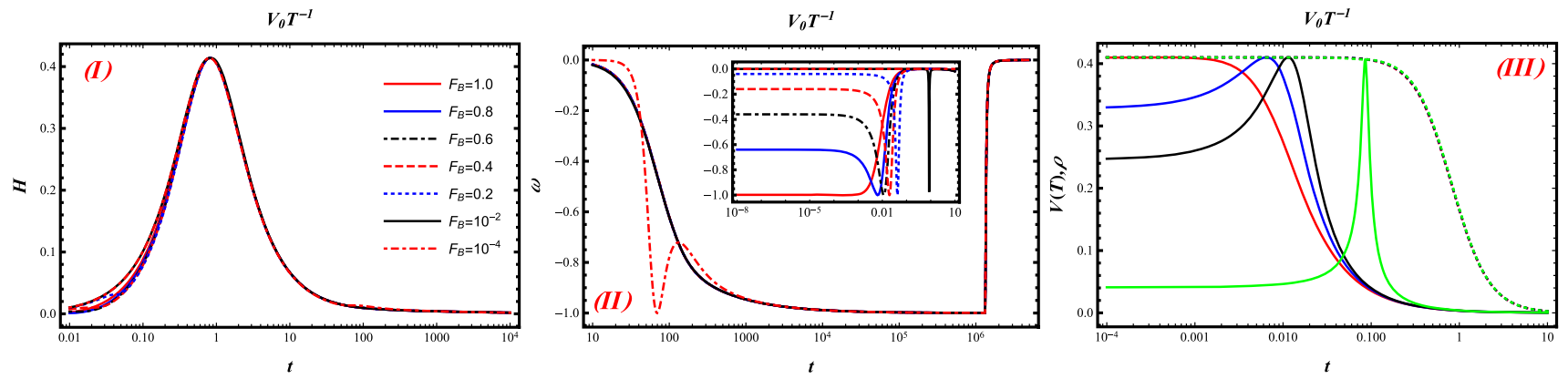

Fig. 2 Evolutions of $H, \omega, V(T)$ and $\rho$ for different initial conditions for an inverse-power potential with $n=-1$. The initial conditions for plots I and II are shown in I. Plot III is of $V(T)$ (solid) and $\rho$ (dotted) for the initial conditions $F_{\mathrm{B}}=1$ (red), $F_{\mathrm{B}}=0.8$ (blue), $F_{\mathrm{B}}=0.6$ (black), and $F_{\mathrm{B}}=0.1$ (green)
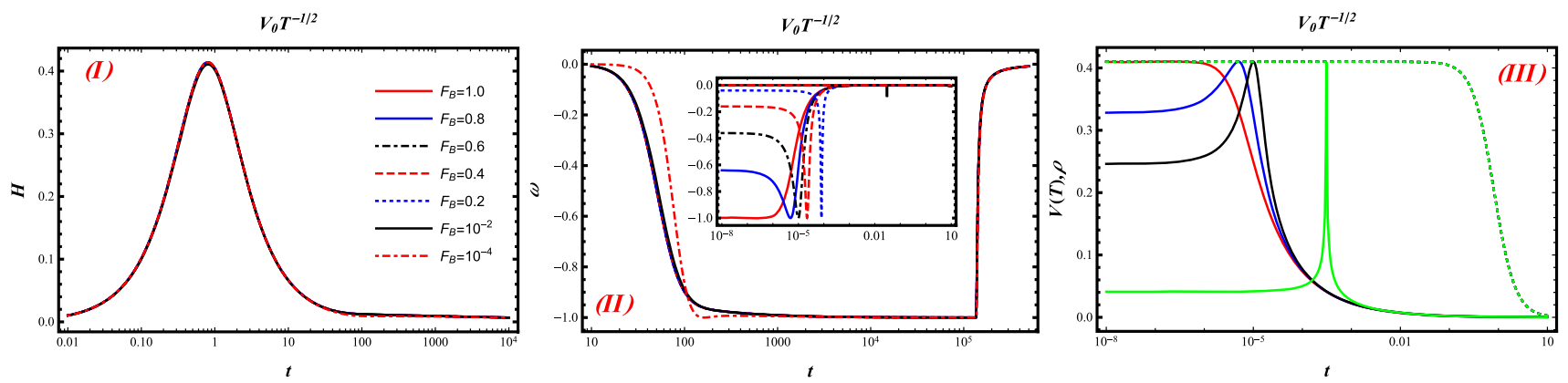

Fig. 3 Evolutions of $H, \omega, V(T)$ and $\rho$ for different initial conditions for an inverse-power potential with $n=-1 / 2$. The initial conditions for plots I and II are shown in II. Plot III is of $V(T)$ (solid) and $\rho$ (dotted) for the initial conditions $F_{\mathrm{B}}=1$ (red), $F_{\mathrm{B}}=0.8$ (blue), $F_{\mathrm{B}}=0.6$ (black), and $F_{\mathrm{B}}=0.1$ (green)

conditions. For the exponential potential, $N \gg 60$ for $F_{\mathrm{B}}=$ 1 and then decreases very quickly to $N=60\left(F_{\mathrm{B}}=7.802 \times\right.$ $\left.10^{-4}\right)$. The numerical analysis reveals that the PED case gives more e-folds when the potential is exponential. However, when the field has an inverse-power potential with either $n=-1$ or $n=-1 / 2$, the e-folds number always more than 60 regardless of the initial conditions.

The numerical analysis and discussion above yield a short conclusion for tachyonic inflation in LQC. Because the efolds number always more than 60 for an inverse-power potential, we will just focus on the exponential potential. When the inflation is driven by a tachyon field with an exponential potential, its main results are as follows.

(1) $F_{\mathrm{B}} \geq 0.6$ : The potential energy dominates at the bounce when the EoS is $\omega_{\mathrm{B}} \leq-0.36$. Because $\dot{T} \leq 0$, the potential decreases during the super-inflation stage until $V \simeq \rho$ (see the third plot of Fig. 1). The duration of super-inflation is quite long $\left(t_{\mathrm{si}}>10^{3}\right.$ Planck seconds) because $\ddot{T}$ and $\dot{T}$ change very slowly. After the superinflation, the Hubble parameter $H$ decreases and the slow-roll conditions are easily met because $\delta_{\mathrm{H}} \ll 1$ and $\epsilon_{\mathrm{H}} \ll 1$ when $\ddot{T}=0$ (at about $10^{4}$ Planck seconds). The slow-roll inflation is very long and $N \gg 60$ in this phase. Although it is possible that $\left|\epsilon_{\mathrm{H}}\right| \ll 1$ and $\eta_{\mathrm{H}} \ll 1$ for a moment during the super-inflation stage, this cannot be considered as a part of the slow-roll inflation phase because $\dot{H}>0$ and $H$ increases, even though $H$ increases very slowly.

(2) $10^{-2}<F_{\mathrm{B}}<0.6$ : The kinetic energy dominates at the bounce when the EoS is in the range $-0.36<\omega_{\mathrm{B}}<$ $-10^{-4}$. The energy density $\rho$ decreases very quickly, and the duration of the super-inflation phase is very short $\left(t_{\mathrm{si}} \sim 1\right.$ Planck seconds). The slow-roll condition is met within $10-50$ Planck seconds after the bounce, and the quantum modification term can be ignored because $\rho \ll \rho_{\mathrm{c}}$. Just as in (1), $N \gg 60$ in this case.

(3) $F_{\mathrm{B}}<10^{-2}$ : The kinetic energy completely dominates at the bounce when $\omega_{\mathrm{B}}>-10^{-4}$. Just as in (2), the duration of super-inflation is very short, and the slowroll condition is met far away from the bounce. The EoS decreases during the damping regime and reaches -1 . The slow-roll condition is met when $\omega<-1 / 3$. However, the duration of $\omega=-1$ depends on the value of $F_{\mathrm{B}}$, and the duration decreases as $F_{\mathrm{B}}$ decreases. When $F_{\mathrm{B}}=7.802 \times 10^{-4}$, the duration is about $4.6 \times 10^{3}$ Planck seconds and $N=60$. When $F_{\mathrm{B}}<7.802 \times 10^{-4}$, the number of e-folds is less than 60 .

The inflation model needs to be consistent with the observational data. In GR scenario, the observation data is difficult to distinguish between canonical scalar field inflation and 
tachyon inflation [45]. The scalar curvature power spectrum (tensor spectrum) in LQC get modified in terms of GR, and it can be written as $P_{\mathrm{R}, \mathrm{h}}=\left(1+\delta_{\mathrm{Pl}}\right) P_{\mathrm{R}, \mathrm{h}}^{\mathrm{GR}}$ with $R(h)$ denoting scalar (tensor) perturbation, and the factor $\delta_{\mathrm{Pl}}$ including the LQC corrections. The tensor-to-scalar ratio reads $r=P_{\mathrm{h}} / P_{\mathrm{R}}$ $[25,26]$, and the holonomy corrections do not contribute to the tensor-to-scalar ratio up to the third order approximation [46]. The tensor-to-scalar ratio is approximately constant in LQC, regardless of the initial state and the adopted quantization approach [49]. In GR, the tensor-to-scalar ratio $r$ can be written in terms of $N[47,48]$,

$r=\frac{16}{2 N+1}$.

The data originated by Planck 2018 show that there is an upper bound on $r, r<0.10$ (Planck TT, TE, EE + lowEB + lensing), or $r<0.064$ (Planck TT, TE, EE + lowE + lensing + BK14) [6]. According to Eq. (17), one finds that a minimum value of the number of e-folds holds, i.e., $N_{\min }=124.5$ for $r<0.064$, and $N_{\min }=79.5$ for $r<0.1$. But the data is still not measured exactly $[6,50]$, and the perturbation theory for tachyonic inflation in the LQC scenario is still absent; we just consider $N_{\min }=60$ in this paper.

\section{Tachyonic inflation probability in LQC}

The equation of motion of the LQC volume variable $v(t)$ and of the tachyonic inflation $T(t)$ can be written as

$\ddot{v}=\frac{3 v}{M_{\mathrm{pl}}^{2}}\left[\rho\left(1-\frac{\rho}{\rho_{\mathrm{c}}}\right)-\frac{1}{2}(\rho+P)\left(1-2 \frac{\rho}{\rho_{\mathrm{c}}}\right)\right]$,

according to Eq. (10).

In this paper, we follow the procedure of Ref. [15] to calculate the tachyonic inflation probability. We consider a phase space $\Gamma$ with quadruplet variables $(v, b ; T, \Pi)$ for $\lambda b \in[0, \pi / 2]$. The Liouville measure of $\Gamma$ is simply $\mathrm{d} \mu_{L}=$ $\mathrm{d} v \mathrm{~d} b \mathrm{~d} T \mathrm{~d} \Pi$. The modified Friedmann equation implies that these variables must lie on a constraint surface $\bar{\Gamma}$ defined by

$\mathscr{C}_{\text {eff }}=-\frac{3}{8 \pi \gamma^{2} \lambda^{2}} v \sin ^{2}(\lambda b)+\frac{1}{v} \sqrt{v^{2} V(T)^{2}+\Pi^{2}} \simeq 0$.

The evolutions of the variables $(v, b ; T, \Pi)$ are

$$
\begin{gathered}
\dot{v}=\frac{3 v}{2 \gamma \lambda} \sin (2 \lambda b), \quad \dot{b}=\frac{4 \pi \gamma \Pi^{2}}{\sqrt{\Pi^{2}+v^{2} V(T)^{2}}}, \\
\dot{T}=\frac{2 \pi \gamma \Pi}{\sqrt{\Pi^{2}+v^{2} V(T)^{2}}}, \quad \dot{\Pi}=-\frac{2 \pi \gamma V(T) V^{\prime}(T)}{\sqrt{\Pi^{2}+v^{2} V(T)^{2}}} .
\end{gathered}
$$

To get the tachyonic inflation probability in LQC, one needs to obtain the Liouville measure of the space $S$ of solu- tions of Eqs. (10) and (19). The Liouville measure $\mathrm{d} \mu$ when pulled back to the surface with constant $b=b_{0}$ is $[15,20,22]$

$\mathrm{d} \hat{\mu}=\sqrt{-V(T)^{2}+\frac{9 \sin ^{4}(b \lambda)}{64 \gamma^{4} \lambda^{4} \pi^{2}}} \mathrm{~d} T \mathrm{~d} v$.

When $\{T(t), v(t)\}$ is a solution to Eq. (21), $\{T(t), \alpha v(t)\}$ (with constant $\alpha$ ) is also a solution. In this case one can fix $v=v_{0}$ in the integrals and choose the value of $v_{0}$ at the bounce point, such as $v_{0}=1$ [17].

The probability of occurrence of an event $E$ can be written as the fractional volume of the region $R(E)$ in $S$ associated with the solutions in which $E$ occurs. Then the probability can be defined as

$P(E)=\frac{\int_{R(E)} \mathrm{d} \hat{\mu}_{L}}{\int_{S} \mathrm{~d} \hat{\mu}_{L}}$.

Considering $\lambda b=\pi / 2$ at the bounce, the probability $P(E)$ of the occurrence of event $E$ is given by

$P(E)=\frac{1}{N} \int_{I(E)}\left[\frac{9}{64 \pi^{2} \gamma^{4} \lambda^{4}}-V(T)^{2}\right]^{1 / 2} \mathrm{~d} T$,

where $I(E)$ is the interval on the $T_{\mathrm{B}}$-axis corresponding to the physically distinct LQC solutions in which $E$ occurs[15]. The quantity $N$ is the total measure of the space $S$ and is given by

$N=\int_{T_{\min }}^{T_{\max }}\left[\frac{9}{64 \pi^{2} \gamma^{4} \lambda^{4}}-V(T)^{2}\right]^{1 / 2} \mathrm{~d} T$,

where $T_{\min }$ and $T_{\max }$ are the minimum and maximum field values allowed by the background. The condition $V(T) \leq \rho_{\mathrm{c}}$ should hold according to the modified Friedmann equation (4). In this case, $T_{\min }=0$ and $T_{\max } \rightarrow \infty$ for the exponential potential. According to Table 1, the exponential potential gives

$$
\begin{aligned}
P_{N \geq 60} & =\frac{\int_{I(E)}\left[\frac{9}{64 \pi^{2} \gamma^{4} \lambda^{4}}-V(T)^{2}\right]^{1 / 2} \mathrm{~d} T}{\int_{T_{\min }}^{T_{\max }}\left[\frac{9}{64 \pi^{2} \gamma^{4} \lambda^{4}}-V(T)^{2}\right]^{1 / 2} \mathrm{~d} T} \\
& =\frac{\int_{7.802 \times 10^{-4} \sqrt{1-F_{\mathrm{B}}^{2}}}^{1} \mathrm{~d} F_{\mathrm{B}}}{\int_{0}^{1} \sqrt{1-F_{\mathrm{B}}^{2}} \mathrm{~d} F_{\mathrm{B}}}=0.999007 .
\end{aligned}
$$

This means that the tachyonic inflation probability is very close to 1 for the exponential potential. As we mentioned in Sect. 3, the bounce is purely KED when $F_{\mathrm{B}}<7.802 \times 10^{-4}$, but this case cannot yield enough e-folds. This is very different from canonical scalar field-driven inflation [20]. Considering Eq. (17), the upper bound on $r$ gives a lower bound 
on $N$, and the probability changes. For example, if $r<0.1$, the probability is $P_{N \geq 79.5}=0.998654(N=79.5$ when $\left.F_{\mathrm{B}}=1.0568 \times 10^{-3}\right)$, and if $r<0.064$, the probability is $P_{N \geq 124.5}=0.997827\left(N=124.5\right.$ when $F_{\mathrm{B}}=$ $1.7063 \times 10^{-3}$ ).

An inverse-power potential with $n=-1$ or $n=-1 / 2$ yields $N>60$ until $F_{\mathrm{B}}=10^{-6}$, as Table 1 and Fig. 3 show. Then $F_{\mathrm{Bmin}}<10^{-6}$, and the inflation probability is greater than 0.99999873 .

We just considered $\dot{T}_{\mathrm{B}}<0$ because the potentials described by Eq. (16) are monotonically decreasing. It is easy to find that the inflation probability is almost the same for $\dot{T}_{\mathrm{B}}>0$ as for $\dot{T}_{\mathrm{B}}<0$.

\section{Conclusion}

A tachyon field acts as an inflaton at a very early time and as cosmic dark matter at a later time. Tachyonic inflation in LQC has been investigated by many researchers [31-33]. Following these researchers, we discussed tachyon field inflation with an exponential potential $V(T) \propto e^{-\alpha T}$ and inversepower potentials of the form $V(T) \propto T^{n}$ with $n=-1$ and $n=-1 / 2$.

As many papers have reported, it is easier to get enough e-folds when the kinetic-energy density dominates at the bounce point when the inflaton is a canonical scalar field [25$28,34]$. However, the tachyon field has no canonical kinetic energy, so the kinetic energy cannot be zero at the bounce. We have found that the potential energy dominates at the bounce when the ratio $F_{\mathrm{B}}$ of the potential to the critical energy density is 1 , and the kinetic energy dominates at the bounce when $F_{\mathrm{B}} \ll 0.5$.

Our numerical analysis found that an exponential potential requires $F_{\mathrm{B}} \in\left[7.802 \times 10^{-4}, 1\right]$ to get enough e-folds, and it is difficult for the pure-KED case to provide enough e-folds. It is very easy to get enough e-folds with an inverse-power potential $(N>260$ for $n=-1$ and $N>333$ for $n=-1 / 2$ when $\left.F_{\mathrm{B}} \geq 10^{-6}\right)$. The tachyonic inflation probability $P(E)$ in LQC depends on $F_{\mathrm{B}} ; P(E)=0.999007$ for an exponential potential, and $P(E)>0.99999873$ for an inverse-power potential with either $n=-1$ or $-1 / 2$.

The probability of tachyonic inflation is very close to 1 , but the probability depends on the mass of the field $[32,33]$. The inflaton mass should be calculated using the power spectrum and the spectral index $[51,52]$. There is still no perturbation theory of tachyon inflation in LQC, so the masses of the potentials are just constants in this paper. On the other hand, the relationship between the tensor-to-scalar ratio and the number of e-folds [see Eq. (17)] does not involve the perturbation theory of tachyonic inflation in LQC; then the results based on Eq. (17) still need to be further addressed, which we hope to do in future work.
Acknowledgements This work is supported by the National Natural Science Foundation of China (Grant No. 11605053), the Hunan Provincial Natural Science Foundation of China (Grant No. 2017JJ3052), and the Research Foundation of Education Bureau of Hunan Province, China (Grant No. 15B060). This work is also supported by the aid program of science and technology innovative research team of Hunan Institute of Technology, and the physics discipline of Hunan Institute of Technology. Mark Kurban from Liwen Bianji, Edanz Editing China (http://www.liwenbianji.cn/ac), edited a draft of this manuscript.

Data Availability Statement This manuscript has no associated data or the data will not be deposited. [Authors' comment: Theoretical predictions can be obtained upon request.]

Open Access This article is licensed under a Creative Commons Attribution 4.0 International License, which permits use, sharing, adaptation, distribution and reproduction in any medium or format, as long as you give appropriate credit to the original author(s) and the source, provide a link to the Creative Commons licence, and indicate if changes were made. The images or other third party material in this article are included in the article's Creative Commons licence, unless indicated otherwise in a credit line to the material. If material is not included in the article's Creative Commons licence and your intended use is not permitted by statutory regulation or exceeds the permitted use, you will need to obtain permission directly from the copyright holder. To view a copy of this licence, visit http://creativecomm ons.org/licenses/by/4.0/.

Funded by SCOAP . $^{3}$

\section{References}

1. A.H. Guth, Phys. Rev. D 23, 347 (1981)

2. A.A. Starobinsky, Phys. Lett. B 91, 99 (1980)

3. A.D. Linde, Phys. Lett. B 108, 389 (1982)

4. A.A. Starobinsky, Phys. Lett. B 117, 175 (1982)

5. V.F. Mukhanov, JETP Lett. 41, 493 (1985)

6. Planck Collaboration, arXiv:1807.06211 [astro-ph.CO]

7. D.H. Lyth, A.R. Liddle, (Cambridge University Press, Cambridge, 2009)

8. I. Agullo, P. Singh, arXiv:1612.01236 [gr-qc]

9. A. Barrau, B. Bolliet, Int. J. Mod. Phys. D 25(08), 1642008 (2016)

10. G. Calcagni, (Springer, Berlin, 2017)

11. A. Ashteka, J. Pullin (eds.), (World Scientific, 2017)

12. C. Rovelli, (Cambridge University Press, Cambridge, 2004)

13. T. Thiemann, (Cambridge University Press, Cambridge, 2007)

14. A. Ashtekar, T. Pawlowski, P. Singh, Phys. Rev. D 74, 084003 (2006)

15. A. Ashtekar, D. Sloan, Gen. Relativ. Gravit. 43, 3619 (2011)

16. A. Corichi, A. Karami, Phys. Rev. D 83, 104006 (2011)

17. L.L. Graef, R.O. Ramos, Phys. Rev. D 98, 023531 (2018)

18. L. Linsefors, A. Barrau, Phys. Rev. D 97, 123509 (2013)

19. L. Chen, J.Y. Zhu, Phys. Rev. D 92, 084063 (2015)

20. A. Ashtekar, D. Sloan, Phys. Lett. B 694(2), 108 (2010)

21. A. Ashtekar, A. Barrau, Class. Quantum Gravity 32, 234001 (2005)

22. A. Bhardwaj, E.J. Copeland, J. Louko, Phys. Rev. D 99, 063520 (2019)

23. B. Bonga, B. Gupt, Gen. Relativ. Gravit. 48(6), 71 (2016)

24. B. Bonga, B. Gupt, Phys. Rev. D 93, 063513 (2016)

25. T. Zhu, A.Z. Wang, G. Cleaver, Phys. Rev. D 96, 083520 (2017)

26. T. Zhu, A.Z. Wang, G. Cleaver, Q. Sheng, Phys. Lett. B 773, 196 (2017)

27. M. Sharma, M. Shahalam, Q. Wu, A.Z. Wang, JCAP 1811, 003 (2018) 
28. M. Shahalam, M. Sharma, Q. Wu, A.Z. Wang, Phys. Rev. D 96, 123533 (2017)

29. M. Shahalam, M. Sami, A.Z. Wang, Phys. Rev. D 98, 043524 (2017)

30. X. Zhang, J.F. Zhang, J.L. Cui, L. Zhang, Mod. Phys. Lett. A 24, 1763 (2009)

31. A. Sen, Phys. Rev. D 74, 043501 (2006)

32. H.H. Xiong, J.Y. Zhu, Phys. Rev. D 75, 084023 (2007)

33. K. Xiao, X.K. He, F. Huang, J.Y. Zhu, Int. J. Mod. Phys. D 23(11), 1450087 (2014)

34. M. Shahalam, M. Sami, A.Z. Wang, Phys. Rev. D 98, 043524 (2018)

35. A. Sen, J. High Energy Phys. 04, 048 (2002)

36. A. Sen, J. High Energy Phys. 07, 065 (2002)

37. A. Ashtekar, T. Pawlowski, P. Singh, Phys. Rev. D 73, 124038 (2006)

38. A. Ashtekar, T. Pawlowski, P. Singh, Phys. Rev. D 74, 084003 (2006)

39. C. Rovelli, E. Eilson-Ewing, Phys. Rev. D 90, 023528 (2014)

40. P. Diener, B. Gupt, P. Singh, Class. Quantum Gravity 31, 105015 (2014)
41. A. Ashtekar, P. Singh, Class. Quantum Gravity 28, 213001 (2011)

42. K.A. Meissne, Class. Quantum Gravity 21, 5245 (2004)

43. M. Domagala, J. Lewandowski, Class. Quantum Gravity 21, 5233 (2004)

44. F. Huang, J.Y. Zhu, K. Xiao, Int. J. Mod. Phys. D 22(06), 1350030 (2013)

45. D.A. Steer, F. Vernizzi, Phys. Rev. D 70, 043527 (2004)

46. T. Zhu, A.Z. Wang, G. Cleaver, K. Kirsten, Q. Sheng, Q. Wu, JCAP 1510, 052 (2015)

47. K. Rezazadeh, K. Karami, S. Hashemi, Phys. Rev. D 95, 103506 (2017)

48. N. Barbosa-Cendejas, J. De-Santiago, G. German et al., JCAP 1803, 015 (2018)

49. L.C. Gomar, G.A. Mena Marugan, D.M. De Blas, J. Olmedo, Phys. Rev. D 96, 103528 (2017)

50. A. Mohammadi, K. Saaidi, H. Sheikhahmadi, Phys. Rev. D 100, 083520 (2019)

51. B. Bolliet, J. Grain, C. Stahl, L. Linsefors, A. Barrau, Phys. Rev. D 91, 084035 (2015)

52. I. Agullo, A. Ashtekar, W. Nelson, Class. Quantum Gravity 30, 085014 (2013) 